\title{
Calidad de vida y reinserción al trabajo en pacientes con trauma moderado y grave por accidentes de tránsito en Medellín (Colombia)*
}

\section{Quality of Life and Reintegration to Work in Patients with Moderate and Severe Trauma due to Traffic Accidents in Medellin (Colombia)}

\section{Qualidade de vida e reinserção no trabalho em pacientes com trauma moderado e grave por acidentes de trânsito em Medellín (Colômbia)}

Fecha de recepción: 17-10-14 Fecha de aceptación: 19-03-15 Disponible en línea: 30-06-15 doi: 10.11144/Javeriana.rgyps18-28.cvrt

Como citar este artículo:

Lugo-Agudelo LH, Salinas-Durán F, Cano-Restrepo BC, Cortés-García D, Hernández-Herrera G, García-García HI . Calidad de vida y reinserción al trabajo en pacientes con trauma moderado y grave por accidentes de tránsito en Medellín (Colombia). Rev. Gerenc. Polít. Salud. 2015; 14(28): 88-96. http:// dx.doi.org/10.11144/Javeriana.rgyps18-28.cvrt

\author{
Luz Helena Lugo-Agudelo** \\ Fabio Salinas-Durán*** \\ Blanca Cecilia Cano-Restrepo**** \\ Diana Cortés-García***** \\ Gilma Hernández-Herrera****** \\ Héctor Iván García-García*******
}

\footnotetext{
Artículo resultado de la investigación Reintegro ocupacional uno y dos años después de accidentes de tránsito moderado y grave en Medellín y el área metropolitana, financiada por la Universidad de Antioquia. Fecha de iniciación: 15 enero del 2012, fecha de finalización: 30 de junio del 2014.

** Médica fisiatra, MSc. en Epidemiología. Grupo Rehabilitación en Salud, Universidad de Antioquia. Correo electrónico: luzh.lugo@gmail.com.

**** Médico fisiatra, Grupo Rehabilitación en Salud, profesor titular de la Facultad de Medicina de la Universidad de Antioquia, Medellín, Colombia. Dirección: Calle 16A Sur \# 45-25, Medellín, Colombia. Correo electrónico: fabios@ une.net.co.

***** Médica fisiatra, especialista en Gerencia de la Salud Ocupacional, Grupo Rehabilitación en Salud, Facultad de Medicina, Universidad de Antioquia. Correo electrónico: blancaceciliacano@gmail.com.

****** Médica fisiatra, Fundación San Vicente. Correo electrónico: dicriscg@yahoo.es.

******* Matemática, MSc. Epidemiología, Grupo Académico de Epidemiología Clínica, profesora de la Facultad de Medicina

$88{ }^{* * * * * * * *}$ Médico, MSc. Epidemiología, Grupo Académico de Epidemiología Clínica y Grupo Rehabilitación en Salud, profesor de la Facultad de Medicina de la Universidad de Antioquia. Correo electrónico: ivan.garcia@udea.edu.co.
} 


\section{Resumen}

Objetivo: evaluar la calidad de vida (Cv) y su relación con la reinserción al trabajo, depresión y dolor, en personas lesionadas en accidentes de tránsito. Métodos: pacientes con trauma moderado o grave, se evaluó reinserción al trabajo, CV (SF-36), depresión (PHQ-9) y dolor (escala análoga visual). Resultados: doce meses después del trauma se reintegraron 367 de 451 pacientes $(81,4 \%)$. Los pacientes no reintegrados tuvieron menores puntaciones en las subescalas de $\mathrm{cv}$, comparados con las de referencia, y en los que sí se reintegraron fueron superiores. No se observó depresión y el PHQ-9 tuvo mayor correlación con la subescala desempeño emocional del sF-36 en los reintegrados y con la de desempeño físico en los que no se reintegraron. La gravedad de la lesión y el dolor afectaron la cv. Conclusión: la cv en pacientes que no se reintegran laboralmente fue inferior y estuvo afectada por la gravedad de la lesión y la depresión.

Palabras clave: calidad de vida; lesiones; accidentes de tránsito; reinserción al trabajo; Escala Análoga Visual del Dolor; PHQ-9

\section{Abstract}

Objective: Assessing the quality of life (cv) and its relationship with reintegration to work, depression and pain in people injured in traffic accidents. Methods: we evaluated reintegration to work, CV (SF-36); depression (PHQ-9); and pain (visual analog scale), in patients with moderate or severe trauma. Results: twelve months after the trauma 367 out of 461 patients reintegrated to work $(81.4 \%)$. The patients that did not reintegrate had lower scores in the $\mathrm{CV}$ subscales, compared to the reference scores; those who did reintegrate to work had higher scores. We did not observe depression and PHQ-9 had a greater correlation with the emotional performance subscale of SF-36 in reintegrated people and with physical performance in those who did not reintegrate. CV was affected by the severity of the injuries and pain. Conclusion: $\mathrm{CV}$ is lower and was affected by the severity of the injuries and depression in patients who did not reintegrate to work.

Keywords: quality of life; injuries; traffic accidents; reintegration to work; visual analog scale for pain; PHQ-9

\section{Resumo}

Objetivo: avaliar a qualidade de vida (cv) e sua relação com a reinserção ao trabalho, depressão e dor, em pessoas lesas em acidentes de trânsito. Métodos: pacientes com trauma moderado ou grave, avaliou-se reinserção ao trabalho, Cv (SF-36), depressão (PHQ-9) e dor (escala analógica visual). Resultados: doze meses depois do trauma reintegraram-se 367 de 451 pacientes $(81,4 \%)$. Os pacientes não reinseridos tiveram menores pontuações nas subescalas de CV se comparados com as de referência, e nos que se reintegraram foram superiores. Não se observou depressão e o PHQ-9 apresentou maior correlação com a subescala desempenho emocional do SF-36 nos reinseridos e com a de desempenho físico nos que não se reinseriram. A gravidade da lesão e a dor afetaram a CV. Conclusão: a CV em pacientes que não foram reinseridos laboralmente foi inferior e esteve afetada pela gravidade da lesão e a depressão.

Palavras-chave: qualidade de vida; lesões; acidentes de trânsito; reinserção ao trabalho; Escala Analógica Visual da Dor; PHQ-9 


\section{Introducción}

En Colombia en el año 2011 se reportaron 181510 accidentes de tránsito (АT), los cuales dejaron 75577 personas heridas y 5528 fallecidas, en tanto que el total de AT en Medellín en el 2012 fue de 46568, los cuales causaron 25232 lesionados (6\% más que en el 2011) y 275 muertos $(1,2)$. En los AT es común que se vean afectados los miembros superiores o inferiores de la persona, lo cual no solo afecta la función, sino que también genera limitaciones en las actividades de la vida diaria y restricciones en la participación, influidas por factores personales y contextuales, de acuerdo con la Clasificación Internacional del Funcionamiento de la Discapacidad y la Salud (CIF) $(3,4)$.

Para la Organización Mundial de la Salud (oMs), la calidad de vida (CV) es "la percepción individual de su posición en la vida en el contexto cultural y de los sistemas de valores en los cuales vive la persona, con relación a sus metas, expectativas, normas y preocupaciones" (5). Esta definición implica que los sujetos evalúan su funcionamiento con referencia a sus expectativas y al logro de determinadas metas. El trauma que se ocasiona en los AT es una causa frecuente de discapacidad laboral y pérdida de días de trabajo, y la reinserción laboral es una de las metas de la rehabilitación, pues el trabajo, aparte de proveer seguridad financiera, estatus social, socialización y posibilidad de autodeterminación, contribuye a la cV. Cuando se analiza la CV como resultado de eventos mórbidos, tales como las lesiones traumáticas, es importante identificar los factores que la determinan y en qué medida son modificables, para poder implementar estrategias de intervención. Los factores modificables son el dolor, la depresión, el estrés y la información que recibe el paciente, y los inmodificables son la edad, el sexo, el mecanismo, el tipo y gravedad de la lesión.
Se ha descrito que después del trauma y de la reintegración al trabajo, en las personas con lesiones graves la cv es menor, en comparación con la de la población general (6).

El objetivo de este estudio fue evaluar la relación existente entre la CV y la reinserción al trabajo, la depresión y el dolor de las personas que tuvieron lesiones moderadas $\mathrm{y}$ graves en AT.

\section{Materiales y métodos}

Se realizó un estudio descriptivo de seguimiento a doce meses con los pacientes del estudio de una cohorte de personas lesionadas en AT en la ciudad de Medellín en los años 2009 y 2010 (7). Los criterios de inclusión fueron: edad entre 16 y 60 años, que vivieran en el área metropolitana de Medellín, haber tenido un trauma moderado a grave de acuerdo con el New Injury Severity Score (NISS), ser atendidos en un servicio de urgencias, que se captaran en el primer mes luego del accidente y que aceptaran participar hasta el seguimiento de dos años, mediante la firma del consentimiento informado. Se excluyeron las personas con trastornos psiquiátricos, con discapacidad cognitiva o con trauma encefalocraneano. Los pacientes se captaron en ocho centros hospitalarios que pertenecen a la red de urgencias de Medellín y son sitios de referencia de víctimas de AT.

En el momento de la inclusión los datos se recolectaron de manera personal e incluyeron aspectos sociodemográficos, afiliación al sistema general de seguridad social, escolaridad, situación laboral y cV. Para el seguimiento se estableció contacto telefónico con algunos y visita personal con otros. Las llamadas y las entrevistas las realizaron auxiliares de investigación del Grupo de Rehabilitación en Salud de la Universidad de Antioquia. 
En Colombia la reinserción al trabajo está definida como la "reincorporación del trabajador al desempeño de una actividad laboral, con o sin modificaciones, en condiciones de competitividad, seguridad y confort, después de una incapacidad temporal o ausentismo, así como también actividades de reubicación laboral temporal o definitiva o reconversión de mano de obra" (8). En este estudio se indagó por algún cambio en la situación ocupacional después del AT. Se consideró reinserción el caso de quienes a los doce meses estuvieran asalariados, trabajaran por su propia cuenta, fueran estudiantes o jubilados que no hubieran tenido una pérdida de capacidad mayor del 50\%, y no reinserción la situación de quienes al año fueran desempleados por motivo de salud u otros motivos y responsables de los quehaceres del hogar.

El dolor se midió con la Escala Análoga Visual (EAv), que va de 0 (no dolor) a 100 (máximo dolor), y se comparó entre los pacientes que se reintegraron y quienes no lo hicieron. La depresión se evaluó con el Cuestionario de Salud del Paciente (PHQ: Patient Health Questionnaire), una herramienta autoadministrada que se emplea para diagnosticar trastornos emocionales. El PHQ-9 es uno de los módulos del PHQ y específicamente evalúa depresión. Este cuestionario, que se encuentra validado en español (9), tiene nueve ítems que son síntomas típicos de depresión (DSM-IV). Las opciones de respuesta van de 0 (nunca) a 3 (casi todos los días), dependiendo de qué tan a menudo la persona ha experimentado estos problemas en las últimas dos semanas. Para calificar la prueba, las respuestas de cada individuo se suman, obteniéndose un puntaje entre 0 y 27 , el cual se categoriza así: síntomas depresivos mínimos: 0-4, leves: 5-9, moderados: 10-14, moderadamente graves: 15-19, y graves: 20-27. Los puntajes mayores de 15 se consideran depresión mayor (10).
La CV se evaluó con el Cuestionario de Salud SF-36, un instrumento que mide la percepción del estado de salud y está validado en Colombia para ser aplicado por entrevista o por el paciente en forma asistida (11). Es una escala genérica que incluye 36 ítems, agrupados en ocho escalas: funcionamiento físico $(\mathrm{FF})$, desempeño físico (DF), dolor corporal (DC), salud general (SG), desempeño emocional (DE), salud mental (SM), vitalidad (VT) y funcionamiento social (FS), y uno adicional: el cambio de la salud en el tiempo (Cs). Los puntajes obtenidos se trasforman en una escala de 0 (peor) a 100 (mejor).

La gravedad de las lesiones se determinó según el NISS, como moderadas aquellas con puntaje entre 4 y 15 y graves cuando este fue mayor de 15 (12).

En el análisis se usaron estadísticas descriptivas, promedio y desviación estándar (DE) o mediana y rango intercuartílico (RIQ) y distribuciones de frecuencias. La distribución normal de las variables numéricas se valoró con la prueba de Kolmogorov-Smirnov. Se realizó comparación de los resultados de los dominios del SF-36, según las variables cualitativas, usando la prueba no paramétrica U de Mann Whitney o Kruskal-Wallis. Para las variables cuantitativas tomadas al año de seguimiento, se calcularon las correlaciones de Pearson o Spearman entre la EAv, los dominios del SF-36 y el PHQ-9 de acuerdo con la reinserción. Para el análisis estadístico se utilizó el programa sPss versión 18 (IBM, Armonk, New York).

\section{Resultados}

Del total de 451 pacientes, $367(81,4 \%)$ se reintegraron luego de doce meses del trauma, y de los $84(18,6 \%)$ que no lo hicieron, $34(40,5 \%)$ explicaban su desempleo por problemas de salud o discapacidad relacio- 
nados con el accidente. Quienes estaban desempleados al año tenían más edad, había más mujeres que hombres, vivían en estratos socioeconómicos bajo-bajo y bajo y su escolaridad era menor de seis años (tabla 1). Con respecto a la gravedad, no se reintegraron a la vida laboral $41(15,7 \%)$ de los 260 pacientes con traumas moderados y $43(22,5 \%)$ de los 191 graves $(\mathrm{p}=0,06)$.

A los doce meses la situación laboral del grupo que se reintegró fue la siguiente: asalariados $219(59,7 \%)$, trabajadores independientes 107 (29,2\%), estudiantes 34 $(9,35 \%)$ y jubilados $4(1,1 \%) .{ }^{1}$ Entre los que se reintegraron, 267 lo hicieron a empleo de tiempo completo (72,8\%), 18 a tiempo parcial $(4,9 \%), 19$ por días $(5,2 \%), 8$ por horas $(2,2 \%), 10$ por producto $(2,7 \%)$ y 4 otros $(1,1 \%)$. De los que no se reintegraron, 16 estaban dedicados al hogar (19\%), 65 desempleados $(77,4 \%)$ y 1 pensionado por invalidez $(1,2 \%)$.

Al ingreso a la cohorte de estudio, los puntajes de CV eran similares, tanto en el grupo de quienes posteriormente se reintegraron laboralmente como en el de quienes no lo hicieron. A los doce meses después del AT, las puntuaciones de todos los dominios de la $\mathrm{CV}$ se incrementaron significativamente en quienes se reintegraron, mientras que en quienes no se reintegraron no mejoró en los dominios: DE, SG, SM y vT. En el grupo que se reintegró, el mayor puntaje se observó en FS, 83,5 (DE $=22,9)$ y el peor en DF, 66,1 (DE $=44,3)$; en el grupo que no se reintegró, el mayor puntaje se observó en cambio de salud, 70,9 (DE = 19,7) y el peor en DF, 34,5 $(\mathrm{DE}=46,6)($ tabla 2).

1 Los jubilados que se incluyeron en el estudio, lo eran por su edad. No hubo pensionados por enfermedad o invalidez, quizá en parte porque el seguimiento no alcanzó tanto tiempo. Es por esto que no aplica la calificación de origen de la invalidez.
La CV en los hombres que se reintegraron un año después fue mejor $(p=0,001)$ en todos los dominios, comparada con la CV inicial. En quienes no se reintegraron, los dominios más afectados fueron DF $(29,1) \mathrm{DE}(46,5) \mathrm{SG}(50,6)$ y DC $(55,2)$. Las mujeres que se reintegraron tuvieron mejor $\mathrm{CV}$ en los dominios DC, DE y DF. Los dominios más afectados en quienes no se reintegraron fueron $\mathrm{DF}(46,9)$, DE $(47,2)$ y DC $(54,5)$ (figura 1$)$.

Las mujeres tuvieron peores desempeños en FF (71,6 vs. 62,0, $\mathrm{p}=0,018), \mathrm{SG}(71,7 v s .63,3$, $\mathrm{p}=0,007)$, sm $(76,0 v s .66,5, \mathrm{p}=0,002)$ y vт (70,1 vs. 60,7, p=0,000), en comparación con los hombres, a pesar de que fue mayor el porcentaje de estos que no se reintegraron.

El dolor (EAv) a los doce meses fue 2,5 (DE = 2,9) en los que se reintegraron, comparado con 4,1 $(\mathrm{DE}=3,4)$ en los que no lo hicieron $(\mathrm{p}<0,01)$. El dolor tuvo una buena correlación con la $\mathrm{CV}$ a los doce meses, tanto en el grupo que se reintegró $(r=0,792)$ como en el que no lo hizo $(r=0,801)$ (tabla 3$)$. Al comparar la depresión a los doce meses en los pacientes que se reintegraron, con respecto a los que no lo hicieron, se observó que fue mínima o leve en 244 (74,2\%) de los que se reintegraron y en $30(38,0 \%)$ de los que no lo hicieron; fue moderada en $71(21,6 \%)$ y $39(49,4 \%)$ y moderadamente grave o grave en $14(4,2 \%)$ y $10(12,7 \%)$, respectivamente.

La correlación entre los puntajes del SF-36 al ingreso y a los doce meses fue mayor en el DE en quienes sí se reintegraron $(r=-0,617)$ y en el DF en los que no lo hicieron $(r=-0,614)$. La correlación más pobre se observó en ambos grupos para el cambio de salud: $r=-0,325$ en los que se reintegraron y $\mathrm{r}=-0,180$ en los que no lo hicieron. La gravedad de la lesión afectó de forma significativa las subescalas del SF-36: FF $(p=0,018)$, sG $(p=0,007)$, sM $(\mathrm{p}=0,002)$ у VT $(\mathrm{p}<0,000)$. 


\section{Discusión}

En el presente estudio se encontró reinserción al trabajo a los doce meses posteriores al Aт en $81,4 \%$ de las personas con lesiones moderadas y graves, y todos los dominios de la $\mathrm{CV}$ fueron significativamente peores en los pacientes que no lograron reintegrarse laboralmente. Además, los dominios de desempeño físico y emocional y función física y dolor corporal fueron los peor puntuados en forma global en el grupo que no se reintegró. Este hallazgo coincide con lo encontrado por Tøien et al. en su estudio de seguimiento a un año en pacientes con trauma, donde los pacientes tenían menores puntajes en estos dominios al ser comparados con la población general (13). En el estudio de Overgaard et al. se encontró un retorno al trabajo o al estudio de $52 \%$ y una mayor $\mathrm{CV}$ entre los que se reintegraron (14). Sin embargo, en el estudio de Moliner et al. no se encontraron diferencias significativas en la $\mathrm{CV}$ entre quienes trabajaban y los que no lo hacían. En el componente físico del sF36 quienes trabajaban tuvieron un puntaje promedio de 40,6 (DE = 5,8) vs. 34,0 (DE = $7,6)$ en los que no lo hacían, en tanto que en el componente mental fue de 42,6 (DE = 10,3) vs. 40,0 ( $\mathrm{DE}=9,9)$, respectivamente. Una de las explicaciones que dan es que los pacientes pudieran haber ajustado sus metas y aspiraciones para reducir la disonancia cognitiva y permitirles adaptarse a una nueva situación vital (15). En el estudio de Gross et al. no se encontraron diferencias en los componentes físico o mental del SF-36 entre quienes tuvieron o no una capacidad reducida para trabajar (componente físico pre 55,8 $[\mathrm{DE}=6,6]$ y $\operatorname{pos} 57,0[\mathrm{DE}=5,6]$, mental pre 49,8 [DE $=12,4]$ y pos $51,4[\mathrm{DE}=$ $10,1]$ ), a pesar de que en esta cohorte $50 \%$ de los pacientes que habían sufrido politraumatismo presentaban una capacidad laboral reducida en el seguimiento a largo plazo (16). Si bien quienes se reintegraron tuvieron una mejor $\mathrm{Cv}$, tomar la reinserción laboral como una variable absoluta y no algo dinámico puede ser complejo, pues los pacientes en un lapso de tiempo pueden tener diferentes vinculaciones laborales, como se encontró en el estudio de Hou et al., con seguimiento a dos años, en el cual $81,2 \%$ de los pacientes tuvieron uno o más reintegros laborales y $38,2 \%$ se mantuvieron reintegrados al final del estudio, pero aquellos que se reintegraron sí tuvieron una mejor Cv (17).

$\mathrm{Al}$ inicio se observaron unos puntajes bajos en los componentes físicos del SF-36, lo cual se correlaciona con la presencia de lesiones en las extremidades, en esta cohorte el trauma ocurrió en los miembros inferiores en 190 $(51,8 \%)$ de los que se reintegraron y $47(56 \%)$ de los que no lo hicieron, y en los miembros superiores en $78(21,3 \%)$ y $13(15,5 \%)$, respectivamente. Este tipo de asociación se ha observado en otros estudios $(18,19)$.

Si se toman como valor de referencia en población sana los puntajes de las subescalas del SF-36 del estudio de García et al., que son: función física 84,1 ( $\mathrm{DE}=2,1)$, desempeño físico 77,4 $(\mathrm{DE}=31,4)$, dolor corporal 74,7 $(\mathrm{DE}=29,4)$, salud general 64,2 $(\mathrm{DE}=22,1)$, vitalidad 65,6 $(\mathrm{DE}=18,1)$, función social $82,4(\mathrm{DE}=23,1)$, desempeño emocional 73,8 $(\mathrm{DE}=33,2)$ y salud mental 65,7 $(\mathrm{DE}=19,2)$ (20), se observa que los pacientes que no se reintegraron, a los doce meses tuvieron unos puntajes de $\mathrm{CV}$ inferiores a los reportados como de referencia, mientras que quienes se reintegraron presentaron puntajes superiores a la media referente en SG, DE y SM. En el primer caso, la cV en el aspecto mental pudiera no mejorar cuando el paciente se da cuenta de que su situación de salud no regresó a los niveles previos que tenía antes del trauma, lo cual favorecería la depresión. En el segundo, se pudiera atribuir en parte al efecto positivo en la percepción que generaría el sobrevivir a un trauma importante 
y poder continuar laborando. Para Kiely et al., la CV mejora en forma constante en los primeros doce a dieiciocho meses luego del trauma, pero posteriormente alcanza una meseta, sin llegar a los valores de referencia de la población (21).

El dolor medido con la EAV tuvo una correlación significativa con la cV en varias de las subescalas del SF-36, pero con mayor valor, como sería de esperarse, en el ítem de DC, tanto en el grupo de quienes se reintegraron como en el de quienes no lo hicieron. Este aspecto enfatiza la importancia que tiene el tratamiento adecuado y oportuno del dolor en los pacientes que han sufrido un traumatismo.

En el presente estudio las subescalas del SF36 que se correlacionaron con la calificación de gravedad del NISs fueron las de FF, SG, SM y VT, lo cual está de acuerdo con el estudio de Lee et al., con 114 participantes, con politraumatismo, la mayoría por Ат y seguidos a tres meses, en el cual la gravedad del trauma (ISS) fue un predictor significativo del puntaje del SF-36 (22). Sin embargo, en el estudio de Kiely et al. no se encontró relación entre la $\mathrm{CV}$, tanto de los dominios físicos como de los dominios mentales, y el puntaje del NISs. La relación entre la gravedad de la lesión y la $\mathrm{CV}$ es inconsistente; hay estudios que se muestran a favor y otros en contra de esto (23-26). Para otros es la percepción subjetiva de gravedad de la lesión, más que el puntaje de la escala NISs, lo que correlaciona con una disminución de los puntajes tanto físicos como mentales de la CV a los seis meses (27).

En el estudio se encontró que las mujeres tuvieron peor desempeño en varios de los ítems del sF-36, hallazgo similar al del estudio de Holbrook et al., quienes también encontraron que las mujeres tienen más riesgo de presentar una peor CV que los hombres luego de un trauma importante, quizá por unos niveles más altos de estrés postraumático, variable que no se midió en este estudio (28). Este hallazgo se observa en otros padecimientos como la enfermedad cardíaca, lo cual se atribuye a la influencia que tienen el apoyo social y la compañía en la forma como la mujer juzga su calidad de vida; además que reportan una mayor dificultad en el componente físico del desarrollo de las actividades de la vida diaria (29). También, en el estudio de Garcia et al. se observó una CV peor en las mujeres que en los hombres, principalmente en las dimensiones de vitalidad, salud general y dolor (30). Sin embargo, en el estudio de Rainer et al., la peor CV se observó en los mayores de 65 años y en hombres (31).

La relación entre el PHQ-9 y el SF-36 fue variable, algo que se ha observado en otros estudios, pues algunos no han encontrado que la gravedad de la depresión fuera un predictor del tiempo para la reinserción al trabajo, pero sí que la depresión es un determinante importante de la $\mathrm{cv}$, y esta a su vez es un predictor importante del tiempo de reinserción, lo que refuerza la importancia de evaluar la Cv y tratar la depresión dentro de un programa integral de rehabilitación posterior a un trauma (32-34).

\section{Limitaciones}

El estudio tiene varias limitaciones: la pérdida del seguimiento por falta de diligenciamiento de algunos formatos; sin embargo, los datos perdidos fueron del $6 \%$ en el peor de los casos. El riesgo potencial de sesgo de selección, pues los pacientes fueron captados de hospitales de alto nivel de complejidad, que suelen contar con personal y equipos más completos para la atención del trauma y pudiera no ser generalizable la conclusión a pacientes que fueran atendidos en hospitales con menores recursos. La relación entre la reinserción laboral y la cv, que puede verse influida por el tiempo transcurrido luego 
del trauma, el estrato socioeconómico y la variabilidad de cada individuo. Además, no se evaluó la presencia de un desorden de estrés postraumático, el cual se presenta entre el 10 y el $40 \%$ de los pacientes luego del trauma y que puede afectar los puntajes mentales del sF-36 (35).

\section{Conclusión}

La $\mathrm{CV}$ en los pacientes que no se reintegraron laboralmente luego de un AT fue peor que en aquellos que sí lo lograron, además, se ve afectada por la gravedad de la lesión y la depresión. Dado que en este tipo de traumas se lesionan frecuentemente las extremidades, lo cual impacta no solo los componentes físicos del sF-36, es importante enfocar los esfuerzos de rehabilitación para tratar de disminuir la discapacidad asociada a este tipo de lesiones, además de tratar el dolor y la depresión que pueden presentar estos pacientes. Asimismo, dado que la mayoría de los pacientes son jóvenes en edad productiva, se deben reforzar las políticas públicas de prevención y la regulación del uso de vehículos.

\section{Financiación}

Esta investigación fue financiada con recursos de la convocatoria CODI de la Universidad de Antioquia (Acta 580 del 23 de julio del 2010) y de la Estrategia de Sostenibilidad 2013-2014.

\section{Agradecimientos}

A la Universidad de Antioquia, a Juliette Weidermann, profesional en gestión de la información, y a la secretaria Eddy Patricia Montoya.

\section{Declaración de conflicto de intereses}

Los autores declaramos que no tenemos conflicto de intereses relacionados con esta investigación.

\section{Referencias bibliográficas}

1. Fondo de Prevención Vial. Anuario Estadístico de Accidentalidad Vial en Colombia 2011 [Internet] [acceso: 14 de junio del 2014]. Disponible en: https://www.fpv.org.co/uploads/documentos/libreria/anuario 2011 pagina.pdf.

2. Secretaría de Transporte y Tránsito, Alcaldía de Medellín. Informe anual de accidentalidad 2012 [Internet] [acceso: 14 de junio del 2014]. Disponible en: http://www.medellin.gov.co/transito/archivos/ accidentalidad/informe_anual_2012.pdf.

3. García HI, Vera CY, Zuluaga LM, Gallego YA. Caracterización de personas lesionadas en accidentes de tránsito ocurridos en Medellín y atendidas en un hospital de tercer nivel 1999-2008. Revista Facultad Nacional de Salud Pública. 2010; 28 (2): 105-17.

4. International Classification of Functioning Disability and Health: ICF. Geneva: World Health Organization; 2001.

5. WHOQOL Group. Development of the WHOQOL: Rationale and Current Status. Int J Mental Health 1994; 23 (3): 24-56.

6. Sluys K, Häggmark T, Iselius L. Outcome and Quality of life 5 Years after Major Trauma. J Trauma 2005; 59 (1): 223-32.

7. Lugo LH, García HI, Cano BC, Arango JC, Alcaraz OL. Multicentric Study of Epidemiological and Clinical Characteristics of Persons Injured in Motor Vehicle Accidents in Medellín, Colombia, 2009-2010. Colombia Médica 2013; 44 (2): 100-7.

8. Colombia. Resolución 2346 del 11 de julio de 2007, por la cual se regula la práctica de evaluaciones médicas ocupacionales y el manejo y contenido de las historias clínicas ocupacionales. Bogotá: Ministerio de la Protección Social; 2007.

9. Baader T, Molina J, Venezian B, Rojas C, Farias R, Fierro C, et al. Validación y utilidad de la encuesta PHQ-9 en el diagnóstico de depresión en pacientes usuarios de atención primaria en Chile. Revista Chilena de Neuro-Psiquiatría 2012; 50 (1): 10-22.

10. Kroenke K, Spitzer R, Williams W. The PHQ-9: Validity of a Brief Depression Severity Measure. JGIM 2001; 16: 606-16.

11. García LH, Lugo LH, Gómez C. Adaptación cultural y fiabilidad del cuestionario de calidad de vida en salud SF-36 en instituciones de salud de Medellín (Colombia) 2002. Revista de la Facultad Nacional de Salud Pública 2006; 24 (2): 37-45.

12. Soberg HL, Bautz-Holter E, Roise O, Finset A. A Long-Term Multidimensional Functional Con- 
sequences of Severe Multiple Injuries Two Years after Trauma: A Prospective Longitudinal Cohort Study. J Trauma 2007; 62 (2): 461-70.

13. Tøien K, Bredal I, Skogstad L, Myhren H, Ekeberg O. Health Related Quality of Life in Trauma Patients. Data from a One-Year Follow Up Study Compared with The General Population. Scand J Trauma Resusc Emerg Med. 2011; 19 (22): 22-34.

14. Overgaard M, Høyer CB, Christensen EF. LongTerm Survival and Health-Related Quality of life 6 to 9 Years After Trauma. J Trauma. 2011 Aug; 71 (2): 435-41.

15. Moliner CE, Durand MJ, Desrosiers J, Coutu MF. Subjective Quality of Life According to Work Status Following Interdisciplinary Work Rehabilitation Consequent to Musculoskeletal Disability. J Occup Rehabil. 2007; 17 (4): 667-82.

16. Gross T, Attenberger C, Huegli RW, Amsler F. Factors Associated with Reduced Longer-Term Capacity to Work in Patients after Polytrauma: A Swiss Trauma Center Experience. J Am Coll Surg. 2010; 211 (1): 81-91.

17. Hou WH, Liang HW, Sheu CF, Hsieh CL, Chuang HY. Return to Work and Quality of Life in Workers with Traumatic Limb Injuries: A 2-Year RepeatedMeasurements Study. Arch Phys Med Rehabil. 2013; Apr; 94 (4): 703-110.

18. McCarthy ML, MacKenzie EJ, Bosse MJ, Copeland C, Hash C, Burgess A. Functional Status Following Orthopedic Trauma in Young Women. J Trauma. 1995; 39 (5): 828-36.

19. Holbrook TL, Anderson JP, Sieber WJ, Browner D, Hoyt DB. Outcome after Major Trauma: Discharge and 6-Month Follow-Up Results from the Trauma Recovery Project. J Trauma. 1998; 45 (2): 315-23.

20. García HI, Vera CY, Lugo LH. Calidad de vida relacionada con la salud en Medellín y su área metropolitana, con aplicación del SF-36. Revista de la Facultad Nacional de Salud Pública. 2014; 32 (1): 26-39.

21. Kiely JM, Brasel KJ, Weidner KL, Guse CE, Weigel JA. Predicting Quality of Life Six Months after Traumatic Injury. J Trauma. 2006; 61 (4): 791-8.

22. Lee BO, Chaboyer W, Wallis M. Predictors of Health-Related Quality of Life 3 Months After Traumatic Injury. J Nurs Scholarsh. 2008; 40 (1): 83-90.

23. Ulvik A, Kvåle R, Wentzel-Larsen T, Flaatten H. Quality of life 2-7 Years after Major Trauma. Acta Anaesthesiol Scand. 2008; 52 (2): 195-201.
24. Harris I, Young JM, Rae H, Jalaludin BB, Solomon MJ. Predictors of General Health after Major Trauma. J Trauma. 2008; 64 (4): 969-74.

25. O'Donnell ML, Creamer M, Elliott P, Atkin C, Kossmann T. Determinants of Quality of Life and Role-Related Disability after Injury: Impact of Acute Psychological Responses. J Trauma. 2005; 59 (6): 1328-34.

26. Harris I, Dao ATT, Young J, Solomon M, Jalaludin BB, Rae H. Factors Predicting Patient Satisfaction Following Major Trauma. Injury 2007; 38 (9): 1102-8.

27. Brasel KJ, Deroon-Cassini T, Bradley CT. Injury Severity and Quality of Life: Whose Perspective is Important? J Trauma. 2010; 68 (2): 263-8.

28. Holbrook TL, Hoyt DB, Anderson JP. The Importance of Gender on Outcome after Major Trauma: Functional and Psychological Outcomes in Women versus Men. J Trauma. 2001; 50: 270-273.

29. Emery C, Frid D, Engebretson T, Alonzo A, Fish A, Ferketish A, et al. Gender Differences in Quality of Life among Cardiac Patients. Phycosomatic Med, 2004; 66: 190-7.

30. García HI, Vera CY, Lugo LH. Calidad de vida relacionada con la salud en Medellín y su área metropolitana, con aplicación del SF-36. Rev Fac Nac Salud Pública 2013; 32 (1): 26-39.

31. Rainer TH, Yeung JH, Cheung SK, Yuen YK, Poon WS, Ho HF, et al. Assessment of Quality of Life and Functional Outcome in Patients Sustaining Moderate and Major Trauma: A Multicentre, Prospective Cohort Study. Injury. 2014; 45: 902-9.

32. Vemer P, Bouwmans CA, Zijlstra-Vlasveld MC, van der Feltz-Cornelis CM, Hakkaart-van Roijen L. Let's Get Back to Work: Survival Analysis on the Return-to-Work after Depression. Neuropsychiatr Dis Treat. 2013; 9: 1637-45.

33. Müller-Tasch T, Peters-Klimm F, Schellberg D, Holzapfel N, Barth A, Jünger J, et al. Depression is a Major Determinant of Quality of Life in Patients with Chronic Systolic Heart Failure in General Practice. J Card Fail. 2007; 13: 818-24.

34. Martin A, Rief W, Klaiberg A, Braehler E. Validity of the Brief Patient Health Questionnaire Mood Scale (PHQ-9) in the general population. Gen Hosp Psychiatry. 2006; 28: 71-7.

35. Holbrook TL, Hoyt DB, Stein MB, Sieber WJ. Perceived Threat to Life Predicts Posttraumatic Stress Disorder after Major Trauma: Risk Factors and Functional Outcome. J Trauma. 2001; 51: 287-92. 